\title{
Surface plasmon coupling on metallic film perforated by two-dimensional rectangular hole array
}

\author{
C. Sauvan, ${ }^{\text {a) }}$ C. Billaudeau, S. Collin, ${ }^{\text {b) }}$ N. Bardou, F. Pardo, and J.-L. Pelouard \\ Laboratoire de Photonique et de Nanostructures (LPN-CNRS), Route de Nozay, 91460 Marcoussis, France \\ P. Lalanne \\ Laboratoire Charles Fabry de l'Institut d'Optique, CNRS, Univ. Paris-Sud, Campus Polytechnique, RD128, \\ 91127 Palaiseau, France
}

(Received 21 September 2007; accepted 10 December 2007; published online 10 January 2008)

\begin{abstract}
The impact of the periodicity on the dispersion relation of surface modes supported by two dimensional (2D) structured metallic films is experimentally and numerically investigated between 1 and $5 \mu \mathrm{m}$. A coupling between modes propagating in two nearly orthogonal directions forming an angle of $77^{\circ}$ is evidenced for a rectangular array of holes perforated in a gold film. The coupling results in a narrow band gap for nonzero wavevectors and in a coupled mode with a large density of states and a strong radiative damping. We show that the gap location in the $(\omega, k)$ space can be precisely tuned by changing the 2D period. (C) 2008 American Institute of Physics.
\end{abstract}

[DOI: $10.1063 / 1.2830333$ ]

Surface plasmons polaritons (SPPs) are surface waves bound to metal/dielectric interfaces that arise from the interaction between light and mobile surface charges. These electromagnetic modes have been known for many years, ${ }^{1}$ but with the recent advances in nanofabrication, there has been a renewed interest in exploiting the optical properties of metals structured at the nanoscale., ${ }^{2,3}$ Introducing subwavelength structures in a metal film modifies the dispersion relation of the flat interface modes ${ }^{4}$ and opens up new possibilities, both for manipulating SPPs on the surface and for coupling them to the far field. Some examples are the enhancement of the molecules fluorescence ${ }^{5}$ or of the light-emitting diodes quantum efficiency ${ }^{6}$ and the coherent emission of light by thermal sources. ${ }^{7}$ All these phenomena are governed by the surface modes supported by the structured metal/dielectric interface and the knowledge of their dispersion relation greatly helps in understanding and engineering the optical properties of structured metallic films.

The dispersion diagram of the structured interface modes can be obtained numerically with three-dimensional fully vectorial calculations ${ }^{8}$ or experimentally with far-field measurements such as angle-resolved reflection or transmission spectra. Until now, such measurements have been performed mainly in the context of the enhanced transmission through subwavelength hole arrays for investigating the role played by SPPs. ${ }^{9-11}$ The surface mode with the lowest energyresponsible for the highest transmission-has been extensively studied, but some other interesting properties of SPPs propagating along perforated interfaces have been less investigated so far. One is the existence of flat plasmonic bands, hence, SPP modes with a large density of states similar to those that can be found in dielectric photonic crystals. ${ }^{12}$ Another one is the influence of the period on the dispersion relation. Indeed, two ingredients exist for the manipulation of light at a subwavelength scale with artificial photonic structures: shape and periodicity. The shape allows to shift the spectral position of resonances ${ }^{11}$ or to create high local

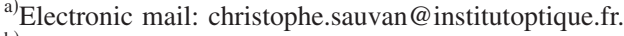

${ }^{b)}$ Electronic mail: stephane.collin@lpn.cnrs.fr.
}

fields, and the array periodicity is responsible for the apparition of band gaps in the dispersion relation. In a twodimensional (2D) array, these scattering phenomena can be rather rich since they combine couplings between counterpropagating SPPs, such as in one-dimensional structures, ${ }^{13}$ and between SPPs propagating along different directions. For square arrays all the coupling phenomena occur at the center or at the edge of the first Brillouin zone, but as we shall see, it is possible to tune the coupling position in the $(\omega, k)$ space with rectangular arrays.

In this letter, we provide experimental evidence of the coupling between SPPs propagating in nearly orthogonal directions on metallic films perforated by subwavelength rectangular hole arrays. This coupling occurs above the dielectric light line for nonzero wavevectors and results in the appearance of a surface mode with a large density of states and a strong coupling to far-field radiation. We have studied a 2D rectangular array of square holes in a gold film deposited on a GaAs substrate (see Fig. 1). The grating periods in the $x$ and $y$ directions are $d_{x}=1 \mu \mathrm{m}$ and $d_{y}=1.25 \mu \mathrm{m}$. The hole width and the gold film thickness are 450 and $250 \mathrm{~nm}$, respectively. The grating has been fabricated by electronbeam lithography and a double lift-off technique described in Ref. 14. This original fabrication process enables the realization of hole arrays with a large area, $2 \times 2 \mathrm{~mm}^{2}$ in this work. Such large dimensions allow to perform far-field optical
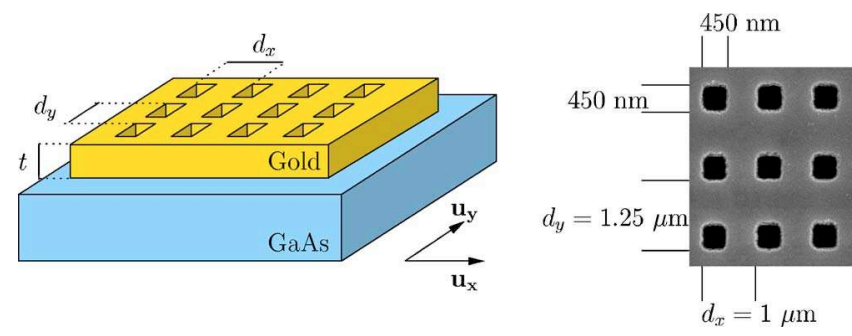

FIG. 1. (Color online) Rectangular subwavelength array of square holes in a gold film deposited on a GaAs substrate. The values of the array parameters are given on the scanning electron microscopy (SEM) picture of the sample. The gold thickness is $t=250 \mathrm{~nm}$. 

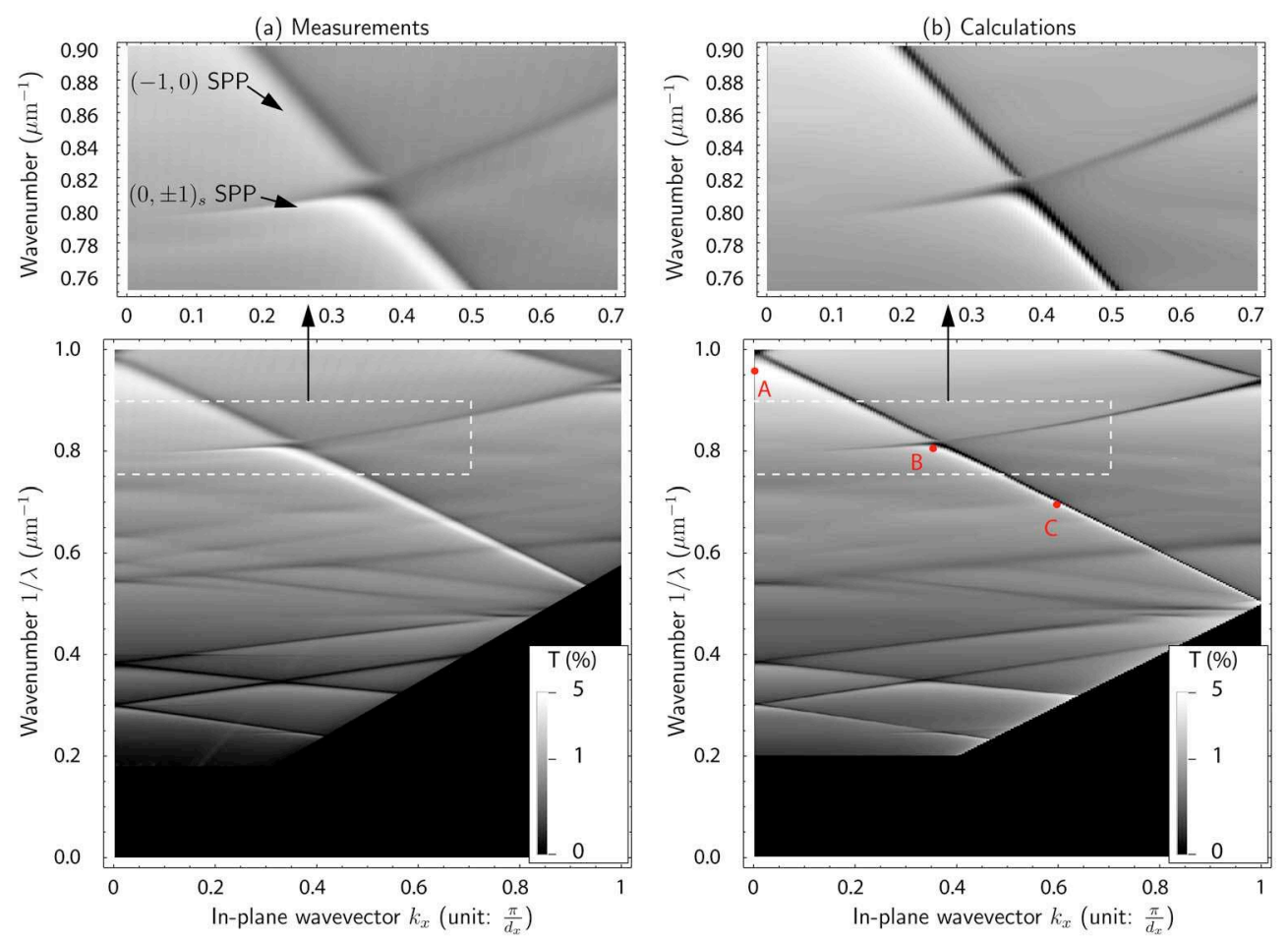

FIG. 2. (Color online) Transmission diagram of the rectangular hole array as a function of $1 / \lambda$ and $k_{x}$ $=k_{0} \sin (\theta)$ for $k_{y}=0$ and $p$ polarization. (a) Experimental data. (b) Numerical results. The insets show an enlarged view of the coupling between the two branches corresponding to surface modes propagating along the air/gold interface in nearly orthogonal directions. measurements with a high angular resolution.

Transmission measurements have been performed with a commercial Fourier transform spectrometer (Bruker Equinox 55). The light is linearly polarized and focused on the sample by using a parabolic and an elliptic mirror. ${ }^{14}$ The incident spot diameter is $1.7 \mathrm{~mm}$ and the beam divergence remains below $\Delta \theta= \pm 0.5^{\circ}$. The transmission measurements are carried out from $\theta=0^{\circ}$ to $\theta=60^{\circ}$ in $0.4^{\circ}$ increments. We use an InSb detector in the $1-5.4 \mu \mathrm{m}$ wavelength range and the spectral resolution is $10 \mathrm{~cm}^{-1}$.

The measured zero-order transmission is represented with a gray log-scale level in Fig. 2(a) as a function of the wavenumber $1 / \lambda$ and the normalized in-plane wavevector in the $x$-direction $k_{x} d_{x} / \pi$. The measurements have been performed in $p$ polarization (incident electric field in the $(x, z)$ plane). The transmission diagram exhibits several bright and sharp Fano-shaped resonances, which are composed of a transmission peak (white lines) associated with a zero transmission (dark lines). For large metal thicknesses, the transmission peaks correspond to the resonant excitation of the leaky surface modes propagating along either the air/gold or the GaAs/gold perforated interface. ${ }^{8}$ Therefore, the white lines in Fig. 2(a) give us directly the different dispersion relations $1 / \lambda=f\left(k_{x}\right)$. The dispersion curves of the air/gold interface modes appear for frequencies above $0.5 \mu \mathrm{m}^{-1}$ and those of the GaAs/gold interface modes appear for lower frequencies because of the high refractive index of GaAs. The transmission minima (dark lines) correspond to a nonresonant effect that has been previously explained through an approximate model. ${ }^{15}$

The most intriguing feature in Fig. 2(a) is the crossing between two bands for $k_{x} \sim 0.35 \pi / d_{x}$ and $1 / \lambda \sim 0.8 \mu \mathrm{m}^{-1}$. A straight band with a linear dispersion relation (negative slope) crosses a flatband with a hyperbolic dispersion relation. The experimental results clearly show the opening of a small stop gap resulting from a coupling between these two surface modes. The coupling point exhibits the highest transmission in the diagram $(>5 \%)$, which means that one of the Downloaded 18 Jun 2009 to 129.175.97.14. Redistribution subject two coupled modes has a strong radiative damping. In Fig. 2(b), we additionally provide a comparison with fully vectorial computational results obtained with a grating solver ${ }^{16}$ that relies on the rigorous coupled wave analysis. ${ }^{17}$ The experimental data are in quantitative agreement with numerical results over the entire transmission diagram. In particular, the rigorous calculations confirm the existence of a coupling between two different surface modes and the opening of a narrow stop gap $(\Delta \lambda \sim 25 \mathrm{~nm})$.

In order to fully understand this coupling phenomenon, we consider the surface modes propagating along a metal interface perforated by a 2D hole array in the limit of infinitely small diameters. The dispersion relation of a SPP propagating on a flat metal/dielectric interface is given by $k_{x}= \pm k_{\mathrm{SPP}}= \pm k_{0}\left(1 / \epsilon_{m}+1 / \epsilon_{d}\right)^{-1 / 2}$, where $\epsilon_{m}$ and $\epsilon_{d}$ are the relative permittivities of the metal and the dielectric, respectively, and $k_{0}=2 \pi / \lambda$ is the free-space wavevector. Clearly, the dispersion relation of the flat interface SPP mode is perturbed by the 2D hole array. It is folded by the array periodicity and altered by the SPP hybridization with the hole modes. ${ }^{4,8}$ In the following, the second effect is neglected and the dispersion relation of the perforated interface modes $(m, p)$ is assumed to be given by

$$
\left(k_{x} \pm m K_{x}\right) \mathbf{u}_{\mathbf{x}}+\left(k_{y} \pm p K_{y}\right) \mathbf{u}_{\mathbf{y}}= \pm \mathbf{k}_{\mathrm{SPP}},
$$

where $K_{x}=2 \pi / d_{x}$ and $K_{y}=2 \pi / d_{y}$ are the components of the grating vector, $m$ and $p$ are two integers, and $\mathbf{u}_{\mathbf{x}}$ and $\mathbf{u}_{\mathbf{y}}$ are the unitary vectors. At the crossing points between different folded branches, and provided that the symmetries of the involved SPPs are the same, a coupling occurs, resulting in the opening of a stop gap between two coupled modes with different frequencies. Equation (1) approximately gives the coupling location in the $\left(\omega, k_{x}, k_{y}\right)$ space but not the coupling strength because the latter is related to the hole shape and size. Such a simple perturbative approach is, therefore, not quantitative but it contains most of the physics related to periodicity effects. In the following, we restrict ourselves to AIP license or copyright; see http://apl.aip.org/apl/copyright.jsp 


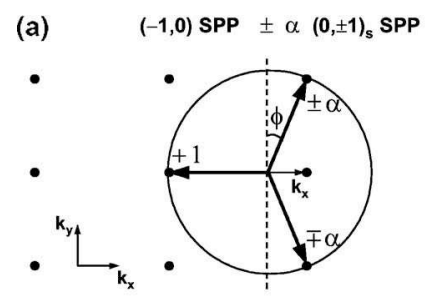

(c)

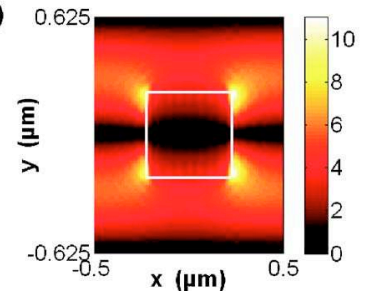

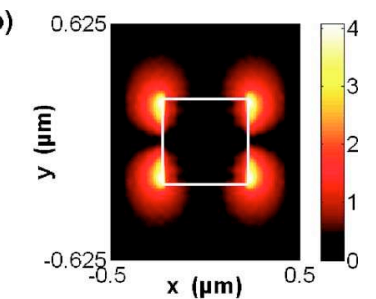

(d)

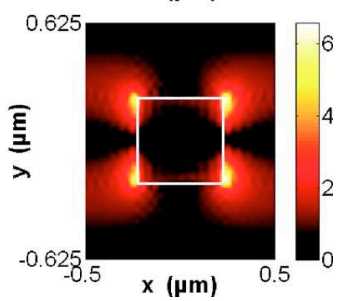

FIG. 3. (Color online) Hybridization of the $(-1,0)$ mode at the coupling point. (a) Schematic representation of the coupled modes. The dots represent the reciprocal lattice shifted by $k_{x}$ and the circle radius is $k_{\mathrm{SPP}}$. The three SPPs involved in the coupling are represented by arrows indicating the propagation direction and the relative weights in the linear combination. [(b)-(d)] Magnetic field distribution $\left|H_{x}\right|$ calculated at the air/gold interface for an incident $p$-polarized plane wave and normalized by the incident magnetic field $\left|H_{\text {yinc }}\right|$, (b) point A in Fig. 2(b) $\left(k_{x}=0,1 / \lambda=0.96 \mu \mathrm{m}^{-1}\right)$, (c) coupling point $\mathrm{B}\left(k_{x}=0.35 \pi / d_{x}, 1 / \lambda=0.8 \mu \mathrm{m}^{-1}\right)$, and (d) point $\mathrm{C}\left(k_{x}\right.$ $\left.=0.6 \pi / d_{x}, 1 / \lambda=0.7 \mu \mathrm{m}^{-1}\right)$.

the surface modes with $k_{y}=0$ in Eq. (1); only these modes are involved in the interpretation of the experimental results shown in Fig. 2.

The stop gap results from a coupling between the $(-1,0)$ SPP with a linear dispersion curve given by $k_{x}-K_{x}$ $=-k_{\mathrm{SPP}}$ and the two $(0, \pm 1)$ SPPs with a flat hyperbolic dispersion curve given by $k_{x}^{2}+K_{y}^{2}=k_{\mathrm{SPP}}^{2}$. For $k_{y}=0$, the $(0,-1)$ and $(0,+1)$ SPPs obey the same hyperbolic dispersion relation and are coupled together through the $2 K_{y} \mathbf{u}_{\mathbf{y}}$ vector of the reciprocal lattice. The resulting coupled modes are $(0, \pm 1)_{s}$ with a magnetic field $H_{y}$ symmetric with respect to the $x$ axis and $(0, \pm 1)_{a}$ with $H_{y}$ antisymmetric. Because of this symmetry difference, the $(0, \pm 1)_{s}$ mode can only be excited with a $p$-polarized incident plane wave, whose $H_{y}$ component is also symmetric with respect to the $x$ axis, whereas the $(0, \pm 1)_{a}$ mode can only be excited in $s$ polarization. ${ }^{14}$ The dispersion curve of the $(-1,0)$ mode crosses both dispersion curves of the $(0, \pm 1)_{s}$ and $(0, \pm 1)_{a}$ modes, but it can only couple to the $(0, \pm 1)_{s}$ mode for the same symmetry reason. The insets in Fig. 2 show a magnified view of this coupling.

Figure 3(a) shows a schematic representation of the two coupled modes that result from a combination of three SPPs propagating in three different directions, one along the $x$ axis and the two other ones along symmetric directions that form a small angle $\phi$ with the $y$ axis given by $\phi=\arctan \left(k_{x} / K_{y}\right)$ $\sim 13^{\circ}$. The expression of the in-plane magnetic field components can be deduced from Fig. 3(a)

$$
\begin{aligned}
& H_{x}=\mp 2 i \alpha H_{0} \exp \left(i k_{x} x\right) \cos (\phi) \sin \left(K_{y} y\right), \\
& H_{y}=H_{0} \exp \left(i k_{x} x\right)\left[\exp \left(-i K_{x} x\right) \pm 2 \alpha \sin (\phi) \cos \left(K_{y} y\right)\right] .
\end{aligned}
$$

In order to show that this representation provides an interpretation of the observed coupling, we have calculated the magnetic field component $H_{x}$ at the air/gold interface for a $p$-polarized incident plane wave and for different wave-

lengths and incident angles. For $k_{x}=0$ [point A in Fig. 2(b)], the incident plane wave excites the $(-1,0)$ mode propagating in the $x$-direction. This mode has a magnetic field oriented purely along the $y$ direction and, therefore, the $\left|H_{x}\right|$ distribution in Fig. 3(b) shows only some localized resonances around the hole edges. At the coupling point B in Fig. 2(b), the magnetic field is a standing wave pattern with a $\sin ^{2}\left(K_{y} y\right)$ variation superimposed on the localized edge resonance [see Fig. 3(c)]. For increasing $k_{x}$ values along the $(-1,0)$ dispersion curve, the standing wave pattern disappears [see Fig. 3(d)]. This result confirms that, for $k_{x}=0.35 \pi / d_{x}$ and $1 / \lambda$ $=0.8 \mu \mathrm{m}^{-1}$, the $(-1,0) \mathrm{SPP}$ mode is coupled to another mode with a magnetic field component $H_{x}$ varying as $\sin \left(K_{y} y\right)$, as shown in Eq. (2). The magnetic field component $H_{y}$ of the $(0, \pm 1)_{s}$ mode has a small amplitude proportional to $\sin (\phi)$. This flat mode with a large density of states is, thus, only weakly coupled to far-field radiation. However, the coupling with the $(-1,0)$ mode creates a coupled mode with a larger $H_{y}$ amplitude [see Eq. (2)] and an enhanced radiative damping (see Fig. 2). This enhancement only exists for rectangular arrays where the coupling occurs for non-zero wavevectors and its location in the $\left(\omega, k_{x}\right)$ space can be tuned by changing the periods $d_{x}$ and $d_{y}$.

In conclusion, we have evidenced a coupling between SPPs propagating in nearly orthogonal directions along a metallic film perforated by a rectangular hole array. Among the two resulting coupled modes, one has a large density of states and a strong radiative damping. This surface mode does not exist in square arrays. Such a periodicity tuning in 2D plasmonic crystals could extend the potential of these periodic subwavelength structures for sensing and light extraction applications.

This work has been partly supported by the European Network of Excellence on Micro-Optics NEMO.

${ }^{1}$ H. Raether, Surface Plasmons on Smooth and Rough Surfaces and on Gratings (Springer, Berlin, 1988).

${ }^{2}$ T. W. Ebbesen, H. J. Lezec, H. F. Ghaemi, T. Thio, and P. A. Wolff, Nature (London) 391, 667 (1998).

${ }^{3}$ S. A. Maier and H. A. Atwater, J. Appl. Phys. 98, 011101 (2005).

${ }^{4}$ J. B. Pendry, L. Martín-Moreno, and F. J. García-Vidal, Science 305, 847 (2004).

${ }^{5}$ H. Rigneault, J. Capoulade, J. Dintinger, J. Wenger, N. Bonod, E. Popov, T. W. Ebbesen, and P. F. Lenne, Phys. Rev. Lett. 95, 117401 (2005).

${ }^{6}$ K. Okamoto, I. Niki, A. Shvartser, Y. Narukawa, T. Mukai, and A. Scherer, Nat. Mater. 3, 601 (2004).

${ }^{7}$ J. J. Greffet, R. Carminati, K. Joulain, J. P. Mulet, S. Mainguy, and Y. Chen, Nature (London) 416, 61 (2002).

${ }^{8}$ P. Lalanne, J. C. Rodier, and J. P. Hugonin, J. Opt. A, Pure Appl. Opt. 7, 422 (2005).

${ }^{9}$ H. F. Ghaemi, T. Thio, D. E. Grupp, T. W. Ebbesen, and H. J. Lezec, Phys. Rev. B 58, 6779 (1998).

${ }^{10}$ W. L. Barnes, W. A. Murray, J. Dintinger, E. Devaux, and T. W. Ebbesen, Phys. Rev. Lett. 92, 107401 (2004).

${ }^{11}$ K. L. van der Molen, K. J. Klein Koerkamp, S. Enoch, F. B. Segerink, N. F. van Hulst, and L. Kuipers, Phys. Rev. B 72, 045421 (2005).

${ }^{12}$ L. C. Andreani and M. Agio, IEEE J. Quantum Electron. 38, 891 (2002).

${ }^{13}$ W. L. Barnes, T. W. Preist, S. C. Kitson, and J. R. Sambles, Phys. Rev. B 54, 6227 (1996).

${ }^{14}$ C. Billaudeau, S. Collin, C. Sauvan, N. Bardou, F. Pardo, and J. L. Pelouard, Opt. Lett. (to be published).

${ }^{15}$ P. Lalanne, C. Sauvan, J. P. Hugonin, J. C. Rodier, and P. Chavel, Phys. Rev. B 68, 125404 (2003).

${ }^{16}$ J. P. Hugonin and P. Lalanne, RETICOLO code for grating analysis, Institut d'Optique, Palaiseau, France (2005).

${ }^{17}$ L. Li, J. Opt. Soc. Am. A 14, 2758 (1997). 